J3eA, Journal sur l'enseignement des sciences et technologies de l'information et des systèmes, Volume 4, Hors-Série 2, 13 (2005)

DOI : http://dx.doi.org/10.1051/bib-j3ea:2005713

(C) EDP Sciences, 2005

L'utilisation pédagogique et l'enseignement des TIC dans les automatismes

B. Riera ${ }^{1}$, F. Gellot $^{1}$, O. Dubois ${ }^{1}$, J.P. Chemla ${ }^{2}$ et S. Triki ${ }^{3}$

${ }^{1}$ Département GCE, IUT Reims-Châlons-Charleville

UFR Sciences Exactes et Naturelles, LAM

Université de Reims Champagne-Ardennes

Moulin de la Housse, BP 1039

F-51687 Reims, France

${ }^{2}$ LUSSI, Polytech' Tours, Université de Tours

7 avenue M. Dassault, F-37200 Tours, France

${ }^{3}$ LAMIH, Université de Valenciennes

Le Mont Houy, BP 311, F-59300 Valenciennes, France 


\title{
L'UTILISATION PEDAGOGIQUE ET L'ENSEIGNEMENT DES TIC DANS LES AUTOMATISMES
}

\author{
Bernard RIERA*, François GELLOT*, Jean-Paul CHEMLA** et Slim TRIKI*** \\ *Département GCE, IUT Reims-Châlons-Charleville, UFR Sciences Exactes et Naturelles, \\ LAM, Université de Reims Champagne-Ardenne, Moulin de la Housse \\ BP1039-51687 Reims. bernard.riera@univ-reims.fr, francois.gellot@univ-reims.fr \\ **Polytech'Tours, 7 avenue M. Dassault \\ 37200 Tours.chemla@univ-tours.fr \\ ***LAMIH, Université de Valenciennes, Le mont Houy \\ BP 311 - 59300 Valenciennes. slim.triki@univ-valenciennes.fr
}

\begin{abstract}
Résumé :
Lors du CETSIS en 1999 [1], les premiers prototypes de partie opérative simulée, appelés Maquettes Virtuelles (MV), avaient été présentés. Les MV sont une représentation fidèle, tant au niveau fonctionnel que comportemental des systèmes habituellement utilisés en Travaux Pratiques (TP). Les MV reposent sur une plateforme initialement développée pour des recherches dans le domaine de la conception et l'évaluation d'outils de supervision adaptés à l'homme [2, 3]. Lors du CETSIS en 2001 [4], nous avions présenté l'architecture choisie pour la nouvelle salle d'API, qui d'une part intégrait les MV et d'autre part permettait une utilisation des API à distance au moyen du réseau Ethernet de l'Université. Les MV, sont actuellement utilisées dans les Universités de Reims, Tours et Valenciennes avant tout pour remplacer les « boites à boutons ». Nous avons aussi réalisé une expérience pédagogique originale de télé-TP dont le bilan est présenté dans cet article. Enfin, nous montrons que la plate-forme développée, compte tenu des technologies utilisées, est devenu un outil pour l'enseignement des TIC dans les architectures d'automatismes.
\end{abstract}

Mots clés : Simulation de partie opérative, Maquettes Virtuelles, télé-TP, supervision, architecture d'automatismes, expérimentation, évaluation

\section{INTRODUCTION}

Lors du CETSIS en 1999 [1], nous avions présenté les premiers prototypes de simulation de partie opérative, appelés Maquettes virtuelles, car ils représentent fidèlement la fonction, la structure et le comportement de systèmes habituellement utilisés en Travaux Pratiques. D'un point de vue technique, le principe a consisté à développer un simulateur auteur (moteur de calculs) et de le connecter à un logiciel de supervision utilisé d'une part pour ses fonctions d'Interfaces Homme-Machine (IHM), et d'autre part pour ses fonctions de communication avec une partie commande (PC) par l'intermédiaire d'une carte de communication. La flexibilité de cette solution permet de simuler ou non la partie opérative (PO) et/ou la partie commande (PC). Ainsi nous avons pu réaliser des expériences de préparation de TP d'automatique continue (régulation du niveau dans 2 cuves couplées) avec une simulation de la PO et de la PC. Il est intéressant de noter que la plateforme avait initialement été développée pour des recherches dans le domaine de la conception et l'évaluation d'outils de supervision adaptés à l'homme [2, 3]. Lors du précédent CETSIS en 2001 [4], nous avions présenté l'architecture choisie pour la nouvelle salle d'Automates Programmables Industrielles (API), qui d'une part intégrait les maquettes virtuelles et d'autre part permettait une utilisation des API à distance au moyen du réseau Ethernet de l'Université. Les maquettes virtuelles ne sont pas pour des raisons pédagogiques évidentes destinées à remplacer les maquettes réelles. Elles sont actuellement utilisées dans les Universités de Reims, Tours et Valenciennes avant tout pour remplacer les «boites à boutons ». Nous avons toutefois réalisé une expérience pédagogique originale de télé-TP qui fait l'objet de cet article. Dans un premier temps les objectifs de l'expérimentation sont détaillés. Ensuite l'environnement technique est présenté. Enfin, les résultats obtenus sont discutés. En guise de conclusion, nous montrons que la plate-forme développée, compte tenu des technologies utilisées, est devenu un outil pour l'enseignement des TIC dans les architectures d'automatismes.

\section{OBJECTIFS DE L'EXPERIMENTATION}

L'objectif principal de cette expérimentation était de réaliser une séance préparatoire de Travaux Pratiques (TP) à distance. Le but du TP (d'une durée de 3 heures) est commander une porte de garage automatique. Pour cela deux étudiants volontaires disposaient du sujet de TP qu'ils avaient lu avant de participer à l'expérimentation. La séance de télé-TP a duré 2 heures. Un binôme (étudiants en $2^{\text {ème }}$ année d'IUP GEII) a participé à cette expérimentation. 


\subsection{Le point de vue « enseignant »}

L'idée de base est de permettre aux étudiants de profiter pleinement de la séance de TP en arrivant bien préparés. D'une façon très générale, une séance de TP d'API vise à transmettre aux étudiants un savoir-faire concernant les outils et les langages de programmation des automates mais surtout la possibilité d'appliquer les concepts théoriques vus en cours pour aboutir à une «bonne» partie commande. Le plus souvent les étudiants doivent se rendre compte de leurs erreurs pour pouvoir assimiler un principe théorique (problèmes de « fond »). Malheureusement, par manque de temps, la séance de TP permet souvent uniquement à l'étudiant d'acquérir un savoir-faire partiel. Cette expérimentation a pour objectif de mettre en évidence s'il est possible ou non à l'étudiant d'acquérir une partie du savoir-faire pendant une séance de télé-TP en vue de se consacrer principalement aux problèmes de «fond»pendant la séance en salle de TP. Pour cela, volontairement la simulation de partie opérative (porte de garage) a été simplifiée pour masquer les problèmes de « fond ». En effet, l'inertie du moteur n'est pas prise en compte. Très souvent les étudiants n'en tiennent pas compte dans leur commande et cela aboutit à un grafcet présentant un problème de stabilité.

\subsection{Le point de vue « étudiant »}

$\mathrm{Si}$ l'on reste objectif, pour une majorité des étudiants, l'objectif principal est de parvenir à bien réussir la séance de TP en vue d'obtenir une bonne note. Cela revient donc à réaliser l'ensemble des consignes décrites dans l'énoncé du sujet de TP dans un temps imparti.

\section{ENVIRONNEMENT TECHNIQUE}

Pour mener à bien cette expérimentation, trois salles distinctes ont été employées et instrumentées de manière spécifique :

- une salle de TP déjà existante, contenant le matériel d'automatisme nécessaire (API, maquettes, ...),

- une salle de travail pour l'étudiant,

- une salle de travail pour l'enseignant.

Les 3 salles sont géographiquement réparties et séparées sur le site du campus. La figure 1 présente l'environnement technique.

\subsection{La salle de TP}

Elle contient tout l'appareillage automatisme, et notamment :

- un API (TSX 57) relié au réseau de l'Université par l'intermédiaire d'un coupleur ethernet,

- un micro-ordinateur de type PC pour la programmation des automates. Il est relié au réseau de l'université et peut aussi dialoguer directement avec l'API par une liaison série RS-485. Ce PC est habituellement utilisé en TP pour programmer en direct l'API au moyen du logiciel PL7 Pro.

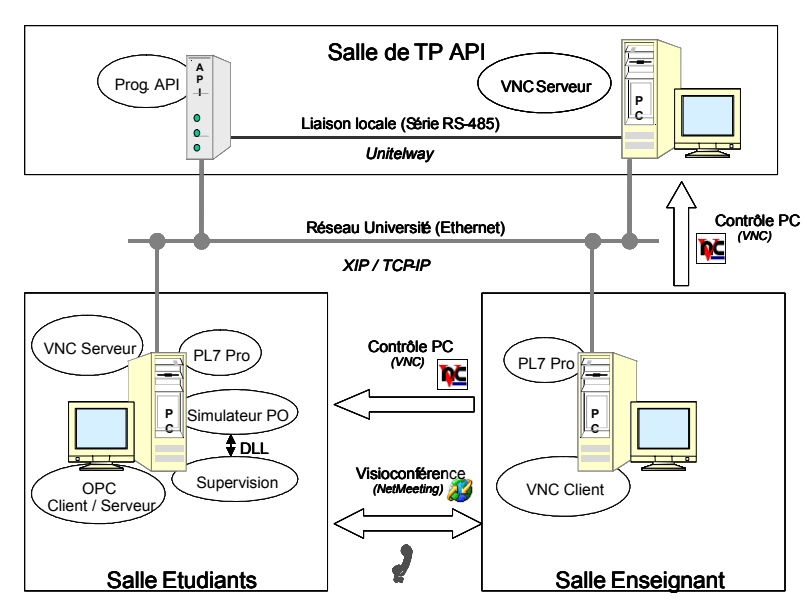

fig 1 : environnement technique

\subsection{La salle « étudiant »}

Elle est équipée d'un PC, relié au réseau de l'Université. Cette machine dispose en interne de :

- un serveur OPC (sur le PC). Le serveur OPC (OLE for Process Control) permet à des applications locales ou distantes (munies d'un client OPC), de communiquer directement avec l'API situé dans la salle de TP.

- PL7 Pro. Il s'agit du module logiciel communiquant avec l'API. Ce logiciel permet d'une part l'écriture du programme API, puis son transfert vers l'API, et enfin le suivi du déroulement du programme. Les étudiants avaient déjà travaillé avec ce logiciel lors de précédents TP d'API. La communication du PC avec l'API se fait au moyen du protocole XIP (Xway sur IP).

- un simulateur de partie opérative (PO). Il s'agit d'un moteur de calcul (simulateur auteur) [2] permettant de simuler, une maquette physique de la salle de TP, tout en en restant pilotable par l'API réel et dialoguant avec le logiciel de supervision (en DDE ou avec une DLL particulière) (cf. figure 2).

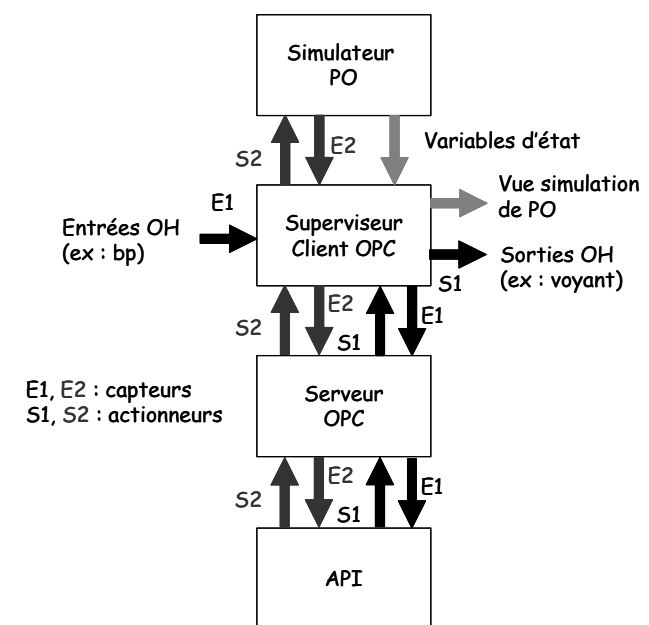

fig 2 : connexions simulateur de PO, superviseur, API Il est important de noter que certains comportements n'ont pas volontairement été simulés. L'idée était de permettre aux étudiants durant la «véritable» séance de TP d'aborder plus rapidement les 
problèmes intéressants d'un point de vue pédagogique.

- un logiciel de supervision qui gère l'affichage graphique de la maquette virtuelle. Le choix est libre. Toutefois, le logiciel de supervision utilisé doit être client OPC pour communiquer avec le serveur OPC et client DDE pour dialoguer avec le simulateur.

\subsection{La salle « enseignant »}

Elle est équipée d'un PC, relié au réseau de l'université, et sur lequel PL7 Pro est présent.

\section{LE DIALOGUE ENSEIGNANT-ETUDIANT}

Dans la suite de l'article, les fonctions notées [a] [b] [c] sont réalisées respectivement au moyen des logiciels suivants :

- [a] NetMeeting

- [b] VNC

- [c] PL7 Pro

\subsection{Côté « étudiant»}

L'étudiant dispose de la possibilité d'interroger l'enseignant à distance pour solliciter des informations complémentaires. Le dialogue, à l'initiative des étudiants ou de l'enseignant se fait au moyen d'une visioconférence [a].

\subsection{Côté « enseignant »}

L'environnement mis en œuvre dans le cadre de cette expérience permet également à l'enseignant de :

- Suivre le travail effectué par l'étudiant sur son poste de travail informatique (visualisation temps réel de l'écran et des actions clavier / souris réalisées) [b].

- Prendre le contrôle à distance du poste étudiant [b].

- Accéder au PC de la salle de TP [b]

- Prendre la main sur l'automate [c]. En effet, il est possible comme cela a été déjà signalé, du PC de la salle de TP de se connecter en direct avec l'API via une liaison série. Cette connexion est prioritaire. Ainsi, il est possible de rattraper à distance les conséquences des erreurs de configuration de l'API commises par les étudiants.

Les PC API et étudiant sont donc des serveurs VNC. Le PC de l'enseignant est client du PC étudiant et du PC de la salle de TP. Les étudiants n'ont pas accès au PC de la salle de TP.

\section{LES RESULTATS OBTENUS}

Les points de vue des 3 acteurs de cette expérimentation ont été étudiés :

- les 2 étudiants ayant participé à l'expérimentation (1 binôme) après le télé-TP, et après la séance de TP réelle,

- l'enseignant du télé-TP,

- l'enseignant du TP réel.
5.1 Le point de vue « étudiant » après le télé-TP Un «brainstorming » avec les étudiants juste après la séance de télé-TP a permis de mettre en évidence, selon leur point de vue, les aspects positifs et négatifs listés ci-après :

\subsubsection{Points positifs}

- Champ de vision toujours libre sur l'écran, pas de gène induite par le professeur. En effet, le plus souvent l'enseignant en TP classique prend physiquement la place de l'étudiant, effectue une manipulation sur le PC tout en expliquant très rapidement aux étudiants qui voient partiellement l'écran.

- Liberté d'actions induite par le fait qu'il n'y a pas de risque de casse.

- Indépendance dans la prise de décisions (les étudiants demandent moins d'aide).

\subsubsection{Points négatifs}

- Problème de communication (qualité du micro, effet de Larsen désagréable)

- Temps de réponse du système.

- Les temps de communication étant importants, en 2 heures, seulement une partie du TP a été réalisée.

- Le nombre de fenêtre est important, il est difficile de visualiser la simulation de partie opérative, les GRAFCET, et la visu avec l'enseignant.

5.2 Le point de vue "étudiant" après la « véritable " séance de TP

Les étudiants ont effectué le TP réel 1 semaine après le télé-TP. Suite à la séance de TP réel, ils ont formulé les remarques suivantes :

\subsubsection{Points positifs}

- La séance de TP s'est très bien déroulée, les étudiants ont réalisé la totalité du TP dans le temps imparti.

- « un homme averti en vaut 2 ! », les étudiants sont arrivés en TP, confiants et ne découvrant pas le sujet.

- La séance préparatoire de télé-TP a été considérée comme bénéfique.

\subsubsection{Points négatifs}

- Les étudiants ont regretté que la simulation ne prenne pas en compte les effets d'inertie du moteur. De ce fait, ils ont été obligés de retravailler leur commande. Ainsi, ce qui était l'objectif recherché par l'enseignant n'a pas été perçu comme tel par les étudiants.

\subsection{Le point de vue « enseignant du télé-TP » 5.3.1 Points positifs}

- Les étudiants étaient concentrés sur leur travail.

- Possibilité de suivre « discrètement» leur activité.

- Les étudiants ont peu contacté l'enseignant et le plus souvent à bon escient.

- L'enseignant a eu un rôle de « hot-liner ». 
- Techniquement tout a fonctionné, et les étudiants ont bien appréhendé le sujet de TP en 2 heures.

\subsubsection{Points négatifs}

- Problème de communication (qualité du micro, effet de larsen désagréable).

- Expliquer à distance est fatiguant, manque d'expérience dans le domaine.

- Difficulté de travailler avec plus d'un binôme.

\subsection{Le point de vue de l'enseignant de TP}

L'enseignant de TP était un ATER. En vue de ne pas influencer son opinion, il n'avait pas été mis au courant de l'expérimentation. Il a trouvé que la séance s'est bien déroulée et que les étudiants ont atteint les objectifs. Toutefois, il convient de noter qu'il s'agit d'un binôme travailleur et performant qui a toujours bien réussi les travaux pratiques.

\section{BILAN}

L'expérience s'est bien déroulée et a donné des résultats intéressants et en particulier :

- il a été mis en évidence que les salles de TP où les étudiants utilisent un PC ne sont pas vraiment adaptées à une interaction enseignant-étudiants performante. En revanche, le télé-TP empêche l'enseignant de se mettre " physiquement » à la place de l'étudiant.

- Les objectifs ont été atteints même si le point de vue des étudiants divergent de celui de l'enseignant (modélisation de l'inertie du moteur).

- Techniquement, tout a fonctionné même si les temps de réponse sont loin d'être négligeables.

Enfin, il s'agit juste d'une expérience dont il n'est pas question de généraliser les résultats obtenus.

La mise en place et l'utilisation de cette plate-forme nous ont conduit à réfléchir à l'enseignement des TIC appliqués aux automatismes.

\section{ENSEIGNEMENT DES TIC DANS L'AUTOMATISME}

Il nous semble que l'enseignement de l'Automatique doit accorder une place aux Technologies de l'Information et de la Communication (TIC) dans les architectures d'automatismes. En effet, les TIC doivent permettre l'établissement de nouveaux modèles de diffusion des données (gestion, production, qualité, maintenance...). L'interconnexion automatisme/informatique est devenue une nécessité dans le mode de fonctionnement des entreprises. L'élaboration d'architectures intégrant le MES (Manufacturing Execution System) et l'ERP (Enterprise Ressource Planning) accroît le besoin de transfert bidirectionnel de données. De ce fait, automatiser aujourd'hui consiste aussi aujourd'hui à accéder à la bonne information au bon moment et à la diffuser à la bonne personne. Les serveurs et clients OPC (OLE for Process Control), très utilisés dans notre plate-forme pédagogique, permettent de faire l'interface entre les différents niveaux de l'entreprise (de la production vers la gestion) et de diffuser les données en provenance du procédé. Il est aussi possible de faire le lien avec des bases de données via les technologies ODBC et OLEDB. La plateforme développée n'est donc plus seulement un outil pédagogique intéressant, elle est devenue un support pour la formation aux TIC appliquées aux automatismes. C'est cette réflexion qui a conduit le département Génie du Conditionnement et de l'Emballage (GCE) de l'IUT Reims - Châlons Charleville en partenariat avec l'UFR Sciences Exactes et Naturelles de l'Université de Reims Champagne - Ardenne, à proposer une licence professionnelle «Supervision et Traçabilité » qui a été habilitée par le ministère pour la rentrée de Septembre 2003.

\section{CONCLUSION}

Dans cet article, une expérience de télé-TP a été présentée et les résultats obtenus détaillés. Il est intéressant de noter comment une application développée initialement en recherche a conduit à des réflexions et à des applications pédagogiques. Le transfert de la recherche vers l'enseignement du thème de la supervision industrielle s'est donc réalisé aussi bien au niveau des outils que des méthodes. Enfin, le télé-TP est amené à se développer car l'enseignement à distance (EAD) [5] est en pleine croissance. Nous insistons toutefois sur l'importance de l'évaluation de ces nouvelles pédagogies.

\section{Bibliographie}

1.Riera, B., Martel, G., Lambert, M. (1999). «Des exemples d'initiation attractive à l'EEA». CETSISEEA'99 ISBN 2854285158 pp 343-346 Montpellier.

2. Riera, B., Martel, G., Lambert, M., Cherifi, E. (1998). « Experimental Platform for Supervision of Complex Automated Systems ", Proceedings of the Seventh IFAC/IFIP/IFORS/IEA on Analysis, Design and Evaluation of MMS, pp. 305-310. Kyoto, Japon.

3. Riera B. « Contribution à la conception d'outils de supervision adaptés à l'homme "), HDR, 14 décembre 2001, Université de Valenciennes.

4. Riera, B., Conreur, G., Chemla, J-P. (2001). « Une salle de TP d'API adaptée aux NTE. » CETSISEEA'01, ISBN 2951752806, pp. 305-308, Université de Clermont-Ferrand.

5. Guillemot, M., Louail, G., Notermann, D., Osete, L., Paulet, D.. (2001). « Enseignement à distance des SED »CETSIS-EEA'01, ISBN 2951752806, pp. 191-194. Université de Clermont-Ferrand. 